\title{
Thomas Harms (Hrsg.): Körperpsychotherapie mit Säuglingen und Eltern. Grundlagen und Praxis
}

\author{
Psychosozial-Verlag, 2016, Gießen, 455 Seiten, 39,90€ (D)
}

T homas Harms versammelt in seinem Werk vieles, was derzeit an Wissen über prä- und postnatale Körperpsychotherapie am Markt ist. Der Verlauf einer Schwangerschaft, das Erleben der Mutter in der Schwangerschaft ebenso wie der Verlauf der Geburt und sogar erlebte oder möglicherweise transgenerational weitergegebene Traumata von Eltern spielen eine wesentliche Rolle für die psychische Konstitution eines Kindes und dessen Eltern.

Das Gemeinsame der vorgestellten Arbeitsmodelle ist der Bezug auf den Körper als wichtigstes Referenzsystem in ihrer psychotherapeutischen Arbeit. Aber es wird deutlich, dass ein multimodales und kreatives Vorgehen unter Einbeziehung von entwicklungsbasierten, familiensystemischen, psychotraumatologischen, bindungstheoretischen und psychoedukativen Zugängen in der Behandlung von Säuglingen und deren Eltern notwendig ist.

Thomas Harms hat eine Kategorisierung der Strömungen in sechs Konzepte bzw. Ansätze versucht, die ihm gut gelungen ist. Das pränatalpsychologische Konzept stellt Ludwig Janus vor: Er beschreibt den Diskurs, in dem dieses Konzept steht, und betont die Wichtigkeit pränatalen Erlebens in der Geschichte dieses Ansatzes und ihrer Vertreter. Er stellt auch heraus, wie wichtig die Selbsterfahrung in Bezug auf die eigene vorgeburtliche und geburtliche Geschichte für TherapeutInnen in diesem Feld ist. Matthew Appelton, Schüler des Pioniers William Emerson, zeigt anhand einer Fallvignette die Folgen eines perinatalen Traumas. Er postuliert, dass Babys mittels der „Babykörpersprache“ und dem „Erinnerungsweinen“ im Gegensatz zum „Bedürfnisweinen“ ihre oft schwierige Geschichte „erzählen“. Regina Bücher, Schülerin des Emerson-Schülers Ray Castillino, beschreibt, wie sie Babys hilft, prä- und peri- natale Erfahrungen zu integrieren. Sie geht salutogenetisch von einer „Original Matrix" als Gesundheitsorientierung aus, mit der sich Klienten letztendlich identifizieren sollen. Auch interessant ist, dass sie Eltern anweist, sehr achtsam zu sein damit, was sie wem über ihr Baby erzählen.

Klaus Käppeli setzt sich in seinem Beitrag intensiv mit der Therapie von Familien nach Kaiserschnittgeburten auseinander. Er beschreibt in berührenden Fallvignetten, wie sich Kinder nach primärem (geplantem) Kaiserschnitt ausgeliefert, überrumpelt und verlassen fühlen. Folgen sind oft Bindungsstörungen, Orientierungsschwierigkeiten, Ängste, Schuldgefühle und vieles mehr. Diesen Kindern fehlt im Leben oft das Gefühl für den richtigen Zeitpunkt. Kindern nach sekundärem Kaiserschnitt (Notkaiserschnitt) fehlt häufig das Selbstvertrauen und die innere Sicherheit, selbstwirksam zu sein. Die Therapie mit diesen Kindern ist Traumaarbeit auf Körperebene: erst Sicherheit und Vertrauen schaffen, dann oft auf Körperebene die gemachten Erfahrungen durcharbeiten und integrieren.

Antonia Stulz-Koller stellt mit einer sehr eindrücklichen Fallgeschichte konkrete psychotherapeutische Interventionen vor, die Babys und Kleinkindern helfen können, Verletzungen, traumatische Erlebnisse und Bindungstraumatisierungen zu überwinden. Sie verwendet Elemente der Pränatalpsychologie, angereichert mit Interventionen aus der systemischen, der narrativen Therapie und dem Kinder-EMDR. Franz Renggli postuliert, dass Babys die Tränen der Eltern weinen, und geht ganz selbstverständlich von traumatisierten Eltern und Großeltern aus, die sich 
in den Körpermustern spiegeln, mit dem Geburtstrauma als Kern.

Dem neoreichianischen Konzept fühlt sich der Herausgeber Thomas Harms zugehörig. In seinem Beitrag zur Emotionellen Ersten Hilfe (EEH) für Säuglinge und Eltern mit den Einsatzgebieten präventive und begleitende Bindungsförderung, Krisenintervention in nachgeburtlichen Krisen und Eltern-Säuglings-Bindungspsychotherapie streift er zunächst die theoretischen Grundlagen der EEH. Er führt das Konzept der Selbstanbindung als Basis für Feinfühligkeit ein: Selbstanbindung meint, dass Eltern über die Fähigkeit einer dualen Aufmerksamkeit verfügen, die zeitgleich auf sie selbst (und die eigene Körperwahrnehmung) und auf ihr Kind gerichtet ist. Die Wiederherstellung der elterlichen Selbstanbindung (und somit auch Selbstregulationsfähigkeit) nennt er als Herzstück der EEH. Danach schildert er Verlauf und Arbeitsweisen der EEH: ein Schreiprotokoll noch vor der ersten Sitzung (auch als Möglichkeit der Distanzierung), Verhaltensbeobachtung und Erkundung der elterlichen Strategien und deren Grenzen im Umgang mit dem Säugling, Fokussierung auf die Körperwahrnehmung der Eltern, die Identifizierung somatischer Marker vor dem Abbruch der Verbindung zum Kind, Körperinterventionen zur Stärkung der elterlichen Bindungsbereitschaft, wie haltgebende Berührung durch den Partner, Atem, imaginative Hilfen zur Stärkung und Sicherung der elterlichen Bindungsbereitschaft, babyzentrierte Körperarbeit. Mechthild Deyringer beschreibt berührend und nachvollziehbar die Schmetterlingsbabymassage und das Konzept „Bindung durch Berührung“, das sie mit Thomas Harms nach Eva Reich weiterentwickelt hat, nicht nur für Babys, sondern eingebettet in Psychotherapie auch für deren Eltern und Erwachsene. Paula Diederichs, die dieses Konzept mitentwickelt hat, hat es noch durch die „Kaiserschnittmassage“ erweitert. Sie fordert Verständnis für die Mütter und deren oft traumatisierenden Geburtserlebnisse ein und behandelt in der von ihr und Thomas Harms konzipierten körperorientierten Krisenbegleitung die ganze Familie, auch wenn Kinder schon drei und mehr Jahre alt sind. Sie stellt ihren Ansatz mit einer Fallstudie dar.

Gerd Poerschke endlich legt den Fokus auf die Väter und stellt das männlich-väterliche Prinzip von Halt vor. Zugleich gibt er wertvolle praktische Anleitungen für die Begriffe Erdung und Zentrierung und die Umsetzung für Väter und ihre Babys. Interessant ist noch die Erwähnung einer Studie, die belegt, dass der Behandlungserfolg einer Eltern-Säuglingstherapie bei Abwesenheit des Vaters deutlich reduziert ist.

Zu den psychoanalytischen Konzepten wird der Ansatz Aucouturier von Christine Hausch vorgestellt. Hier geht es um die Arbeit im Psychomotorik-Raum, die vom frühen tonisch-emotionalen Erleben des Kindes ausgeht. Doris Lange stellt die körperpsychotherapeutische Methode der funktionellen Entspannung vor, die mit dem Grundkonzept der psychosomatischen Selbstregulation arbeitet, das sie von Marianne Fuchs gelernt hat. Hier wird die körperorientierte Methode mit dem Konzept des Übertragungserlebens verbunden. Eine entscheidende Frage ist die Selbstwahrnehmung der Therapeutin bzw. des Therapeuten.

Petra Saltuari beschreibt detailliert einen Therapieprozess über zwölf Stunden mit einer Frequenz von vier Stunden pro Woche, den sie im Rahmen ihrer Arbeit mit Schwangeren, die erheblich psychisch belastet sind, durchgeführt hat. Auch sie arbeitet mit Funktioneller Entspannung, kombiniert mit Kunsttherapie.

Einen interaktionalen Ansatz und videogestützte Konzepte stellt Peter Geißler vor, der als Vertreter der analytischen Körperpsychotherapie seinen Ansatz als Erweiterung analytischer Psychotherapie verstanden wissen will. Er analysiert detailliert eine 2-minütige Sequenz einer Mutter-Kind-Interaktion und beschreibt, wie erst durch wiederholte Analyse, teilweise in Zeitlupe, Dinge sichtbar werden, mit besonderem Fokus auf Körperabstimmungen und nonverbale Interaktionen.

Tanz- und bewegungstherapeutische Ansätze kombiniert mit psychoanalytischen und interaktionalen Konzepten stellt Sabine Trautmann-Voigt mit dem Bonner Modell der Interaktionsanalyse 
vor, das ein mehrdimensionales psychodynamisches Diagnostikinventar darstellt unter Einbeziehung von Videoanalysen der Sitzungen. Sie setzt sich kritisch mit den ihrer Meinung nach zu schnell und von fachfremden Berufsgruppen diagnostizierten „Bindungsstörungen“ auseinander. Einen ganz anderen Ansatz der somatischen Bewegungsarbeit stellt Thomas Greil mit dem Body Mind Centering nach Bonnie Bainbridge Cohen vor. Hier wird die Bewegungsentwicklung nach einem bewegungstherapeutischen Konzept gefördert, das sich durchaus in vielem deckt mit den vorher vorgestellten Methoden - auch hier gibt es Haltetechniken, Geburtsbewegungssimulationen, ein „Lesen“ der Bewegungen der Babys und der Eltern.

Unter Sonstigen Ansätzen hat Thomas Harms die craniosacrale Osteopathie, die Rudolf Merkel vorstellt, und die systemische Hörtherapie nach Tomatis, erläutert von Dirk Beckedorf, subsumiert. Die Craniosacraltherapie hat vermutlich Eingang in diesen Band gefunden, weil sie Säuglingen bzw. deren Eltern häufig empfohlen wird und KörperpsychotherapeutInnen auch oft Hand in Hand mit ihr arbeiten. Sie geht von der Existenz einer autonomen rhythmischen Bewegung von Hirn und Hirnwasser aus, die eine Eigenbewegung haben und von erfahrenen Therapeutlnnen am Cranium und am ganzen Körper mit den Händen wahrnehmbar sind. Dies ist aber kein Ersatz für Physiotherapie, Logotherapie, Psychotherapie, Ergotherapie usw.
Dirk Beckedorfs Text beschreibt die systemische Hörtherapie nach Tomatis. Mittleren Tonbereichen werden Sicherheit und Bindung zugeschrieben, höheren Frequenzen Stress, und sehr tiefe Töne sind mit Angst und Lähmung assoziiert. In der Hörtherapie hören Kinder und Erwachsene Mozart und gregorianische Gesänge, die über spezielle Kopfhörer mit Luft- und Knochenleitung vermittelt werden. Sehr spannend ist die Erforschung des pränatalen Hörens, die belegen, dass es auch schon in sehr frühen fötalen Stadien eine Form von Hören gibt. Deshalb wird häufig auch die aufgenommene Stimme der Mutter eingesetzt, die den Kindern mit einem Filter von 8000 Hertz vorgespielt wird - was die Kinder an das Knochenhören in den ersten Schwangerschaftsmonaten erinnern soll. Das löst ein deutliches Bindungsverhalten aus, die Kinder werden ruhiger, regressiver und suchen Nähe und Bindung.

Abgerundet wird dieses Buch durch ein fundiertes Plädoyer über die Sinnhaftigkeit, in frühe Hilfen zu investieren. Insgesamt ein äußert spannendes Buch für alle Therapeutlnnen, die mit Säuglingen und jungen Familien arbeiten, auch wenn sie keinen körperpsychotherapeutischen Schwerpunkt haben.

\section{Christiane Degenhardt}

DOI 10.2378/ ktb2017.art17d 